\title{
ANÁLISE DE ESTRUTURAS MULTICAMADAS UNIAXIAIS
}

\author{
Lucio Cividanes
}

\author{
Instituto Nacional de Pesquisas Espaciais \\ Divisão de Eletrônica Aeroespacial \\ 12001-970, S. José dos Campos, SP - Brasil \\ lucio@dea.inpe.br
}

\author{
A.V. Proaño De la Torre \\ Escuela Politécnica del Ejército \\ Centro de Investigación Cientifica y Tecnológica \\ Sangolquí - Ecuador \\ anatorre@1i.fie-espe.edu.ec
}

\author{
J.C. da S. Lacava \\ Instituto Tecnológico de Aeronáutica \\ Divisão de Engenharia Eletrônica \\ 12280-900, S. José dos Campos, SP - Brasil \\ lacava@ele.ita.cta.br
}

\begin{abstract}
Resumo - Utilizando o formalismo de Fourier, este trabalho descreve um modelo para análise de estruturas multicamadas uniaxiais. São determinados os campos eletromagnéticos nas diversas camadas dessas estruturas, bem como as respectivas funções de Green espectrais. Resultados numéricos são apresentados para a antena de microfita e para a linha de fita que alimenta uma fenda retangular.

Abstract - This paper presents a full-wave analysis method for uniaxial multilayered structures. Working in the Fourier domain, expressions for the transformed electromagnetic field components are derived. Consequently, spectral Green's functions in compact and closed forms are obtained. Numerical results for microstrip antennas and for stripline structures are presented.
\end{abstract}

Palavras-chave: Estruturas multicamadas, anisotropia uniaxial, domínio espectral, método dos momentos, antenas de microfita, linhas de fita.

\section{INTRODUÇÃO}

A análise dos campos eletromagnéticos em estruturas planas multicamadas, excitados por fontes imersas nessas camadas, tem aplicações em vários campos da engenharia eletromagnética tais como optoeletrônica, circuitos de microondas e antenas [1]. Em geral, uma ou mais dessas camadas podem ser anisotrópicas. Essas anisotropias podem ser naturais. como as exibidas pelos materiais cristalinos [2], ou mtrouuzidas pelos processos de fabricação, ou ainda provocadas artificialmente com o objetivo de melhorar o desempenho de circuitos e antenas [3].

Neste trabalho é apresentado um modelo para a análise de estruturas multicamadas com anisotropias uniaxiais. Nesse modelo, os campos eletromagnéticos nas diversas camadas são determinados empregando-se a transformada dupla de Fourier, que encaminha a solução do problema para o domínio espectral. En conseqüência, são determinadas as funções de Green espectrais, o que possibilita uma simplificação considerável da aplicação do método dos momentos, uma vez que essa abordagem efetivamente remove as singularidades apresentadas pelas funções de Green espaciais [4]. A versatilidade da estrutura em estudo permite analisar várias geometrias de interesse do setor aeroespacial. Dessas, neste trabalho serão analisadas a antena de microfita retangular alimentada por uma prova coaxia] e a linha de fita que alimenta uma fenda retangular.

\section{TEORIA}

A Figura 1 apresenta a geometria da estrutura multicamadas a ser estudada. Esta estrutura é composta por $N$ camadas dielétricas lineares, homogêneas e anisotrópicas (anisotropias do tipo uniaxial). Dessas, $T$ camadas estão confinadas entre os planos condutores perfeitos $z=-d_{1}$ e $z=0$. As outras $N-T$ camadas estão localizadas entre o plano $z=0 \mathrm{e}$ o espaço livre $\left(z>d_{N}\right.$, permissividade elétrica $\varepsilon_{0}$ e permeabilidade magnética $\mu_{0}$ ). No plano de terra $z=0$ existe uma abertura que acopla as camadas confinadas com as camadas localizadas na região $d_{N}>z>0$. Cada camada uniaxial é caracterizada por uma permissividade elétrica tensorial $\tilde{\varepsilon}_{n}$

e por uma permeabilidade magnética escalar $\mu_{0}$. Em cada interface não condutora encontra-se impressa uma superfície $S_{n}$ perfeitamente condutora de espessura infinitesimal, sobre a qual é definida uma densidade de corrente elétrica superficial $\zeta_{n}(x, y)$. Dessa forma, a estrutura torna-se bastante versátil, pois permite a análise de várias configurações de interesse.

Os campos eletromagnéticos nas várias camadas anisotrópicas e no espaço livre serão determinados considerando-se a estrutura como um problema de contorno onde as densidades de corrente superficiais e a componente tangencial do campo elétrico, localizada no plano da abertura, são as fontes virtuais desses campos. As equações de onda nas diversas camadas anisotrópicas e no espaço livre serão resolvidas no domínio de Fourier [5]. 


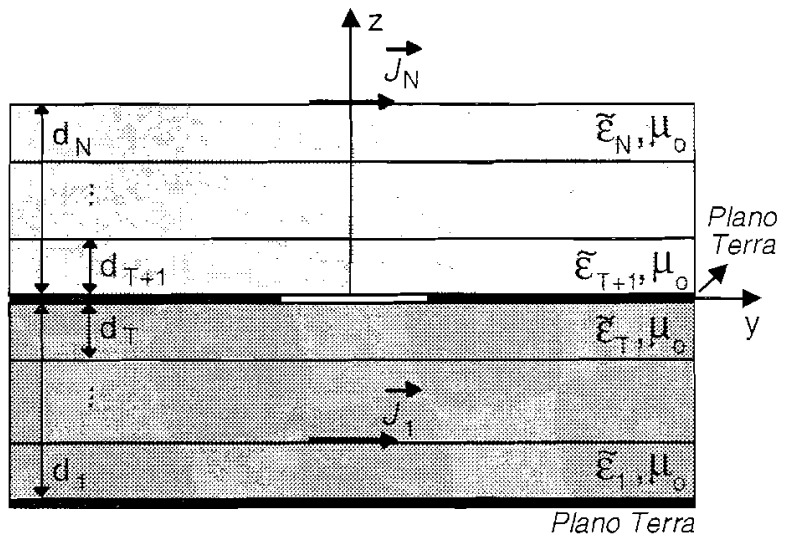

Figura 1. Geometria da estrutura multicamadas uniaxial.

\section{CAMPOS NAS CAMADAS UNIAXIAIS}

Nesta seção são determinadas as expressões para os campos eletromagnéticos no interior da n-ésima camada anisotrópica. No caso de variações harmônicas da forma $e^{i \omega t}$, as equações de onda para meios sem fontes são:

$$
\begin{aligned}
& \nabla \times\left[\widetilde{\varepsilon}_{n}^{-1} \cdot \nabla \times \stackrel{\rho}{H}_{n}(x, y, z)\right]-\omega^{2} \mu_{0} H_{n}(x, y, z)=0 \\
& \nabla \times \nabla \times E_{n}(x, y, z)-\omega^{2} \mu_{0} \widetilde{\varepsilon}_{n} \cdot \rho E_{n}(x, y, z)=0
\end{aligned}
$$

com a seguinte representação matricial para o tensor permissividade (eixo óptico ao logo do eixo z):

$$
\tilde{\varepsilon}_{n}=\left[\begin{array}{ccc}
\varepsilon_{x n} & 0 & 0 \\
0 & \varepsilon_{x n} & 0 \\
0 & 0 & \varepsilon_{\Sigma n}
\end{array}\right] .
$$

Se perdas forem consideradas, os elementos $\varepsilon_{n !}$ e $\varepsilon_{n n}$ do tensor deverão ser complexos.

Operando no domínio de Fourier, são obtidas as seguintes equações diferenciais para as componentes do campo elé-trico transformado:

$$
\begin{aligned}
& {\left[D_{z}^{4}+A_{2} D_{z}^{2}+A_{4}\right] \varepsilon_{\eta n}\left(k_{x}, k_{y}, z\right)=0} \\
& {\left[D_{z}^{2}-A_{3}\right] \varepsilon_{z n}\left(k_{x}, k_{y}, z\right)=0}
\end{aligned}
$$

onde

$$
\begin{aligned}
& A_{2}=-\left(u^{2}-\omega^{2} \mu_{0} \varepsilon_{x n}\right)-\varepsilon_{x n} \varepsilon_{z n}^{-1}\left(u^{2}-\omega^{2} \mu_{0} \varepsilon_{z n}\right) \\
& A_{3}=\varepsilon_{x n} \varepsilon_{z n}^{-1}\left(u^{2}-\omega^{2} \mu_{0} \varepsilon_{z n}\right) \\
& A_{4}=\varepsilon_{x n} \varepsilon_{z n}^{-1}\left(u^{2}-\omega^{2} \mu_{0} \varepsilon_{x n}\right)\left(u^{2}-\omega^{2} \mu_{0} \varepsilon_{z n}\right) \\
& u^{2}=k_{x}^{2}+k_{y}^{2}
\end{aligned}
$$

e $\eta=x$ ou $y$.

Soluções para as equações transformadas (3) e (4) podem ser escritas como:

$$
\varepsilon_{\eta n}\left(k_{x}, k_{y}, z\right)=\sum_{p} \mathbf{e}_{\eta p n} e^{i \gamma_{p \eta}=}
$$

$$
\varepsilon_{z n}\left(k_{x}, k_{y}, \bar{s}\right)=\sum_{q} \mathbf{e}_{z q n} e^{i \gamma_{q n}=}
$$

onde $\mathbf{e}_{\eta p}=\mathbf{e}_{\eta p n}\left(k_{x}, k_{y}\right)$ são funções a serem determinadas,

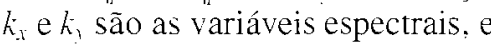

$$
\begin{aligned}
& \gamma_{1 n}=\sqrt{\omega^{2} \mu_{0} \varepsilon_{x n}-u^{2}} \\
& \gamma_{2 n}=\sqrt{\varepsilon_{. n n} \varepsilon_{-n}^{-1}\left(\omega^{2} \mu_{0} \varepsilon_{-n}-u^{2}\right)} \\
& \gamma_{3 n}=-\gamma_{1 n} \\
& \gamma_{4 n}=-\gamma_{2 n}
\end{aligned}
$$

$\operatorname{com} p=1,2,3$ e 4 e $q=2$ e 4

De modo análogo. são obtidas as seguintes expressões para as componentes do campo magnético transformado:

$$
\begin{aligned}
& \#_{\eta n}\left(k_{x}, k_{y}, z\right)=\sum_{p} \mathbf{h}_{\eta p n} e^{i \gamma_{p n} z} \\
& \#_{z, n}\left(k_{x}, k_{y}, z\right)=\sum_{r} \mathbf{h}_{z m} e^{i \gamma_{m n} z}
\end{aligned}
$$

onde $\mathbf{h}_{\eta p n}=\mathbf{h}_{\eta p n}\left(k_{x}, k_{y}\right)$ e $r=1$ e 3 .

Neste ponto é importante salientar que, em cada camada anisotrópica. são desconhecidas as funções $\mathbf{e}_{\eta p n}$ e $\mathbf{h}_{\eta p m}$. em um total de vinte incógnitas. As expressões para os campos eletromagnéticos no domínio espacial são obtidas das transformadas inversas de $\varepsilon_{n}^{\mu}\left(k_{x}, k_{y}, z\right)$ e $\frac{\mu_{k}}{z_{n}}\left(k_{x}, k_{y}, z\right)$. Para a $\eta$-ésima componente do campo e a $p$-ésima solução de $\gamma_{n}$, tem-se:

$$
\begin{aligned}
& E_{\eta p n}=\frac{1}{4 \pi^{2}} \int_{-\infty}^{+\infty} \int_{\eta p n} e^{-i\left(k_{x} x+k_{y} y-\gamma_{p n} z\right)} d k_{x} d k_{y} \\
& H_{\eta p n}=\frac{1}{4 \pi^{2}} \int_{-\infty}^{+\infty} \int_{\eta p n} \mathbf{h}^{-i\left(k_{x} x+k_{y} y-\gamma_{p n} z\right)} d k_{x} d k_{y}
\end{aligned}
$$

onde, para simplificar a nomenclatura, fez-se $E_{\eta p n}=$ $E_{\eta j n}(x, y, z)$ e $H_{\eta p n}=H_{\eta p n}(x, y ; z)$.

Relações úteis entre as funções $\mathbf{e}_{\eta p m}$ e $\mathbf{h}_{\eta p m}$ são obtidas introduzindo as expressões para as componentes dos campos eletromagnéticos, equações (17) e (18), nas equações de Maxwell para os rotacionais dos campos elétrico e magnético, resultando em:

$$
\begin{aligned}
& \mathbf{e}_{x p n}=u^{-2}\left[\varepsilon_{z n} \varepsilon_{x n}^{-1} k_{x} \gamma_{q n} \mathbf{e}_{z q n}-\omega \mu_{0} k_{y} \mathbf{h}_{z m}\right] \\
& \mathbf{e}_{y p n}=u^{-2}\left[\varepsilon_{z n} \varepsilon_{x n}^{-1} k_{y} \gamma_{q n} \mathbf{e}_{z q n}+\omega \mu_{0} k_{x} \mathbf{h}_{z m}\right] \\
& \mathbf{h}_{x p n}=u^{-2}\left[\omega \varepsilon_{z n} k_{y} \mathbf{e}_{z q n}+k_{x} \gamma_{m} \mathbf{h}_{z m}\right] \\
& \mathbf{h}_{y p n}=u^{-2}\left[-\omega \varepsilon_{z n} k_{x} \mathbf{e}_{z q n}+k_{y} \gamma_{m} \mathbf{h}_{z m}\right]
\end{aligned}
$$

Observe que esta última operação reduziu drasticamente o número de incógnitas em cada camada anisotrópica. Somente as funçōes $\mathbf{e}_{-q n}$ e $\mathbf{h}_{z ! n}$ são desconhecidas, ou seja, quatro incógnitas em cada uma das camada. 


\section{CAMPOS NO ESPAÇO LIVRE}

Usando um procedimento similar ao utilizado nas camadas anisotrópicas, são obtidas as seguintes expressões para as componentes dos campos eletromagnéticos transformados na região de espaço livre:

$$
\begin{aligned}
& \varepsilon_{\eta_{0}}\left(k_{x}, k_{y}, z\right)=\mathrm{e}_{\eta_{0}} e^{-i \gamma_{0} z} \\
& \boldsymbol{z}_{\eta_{0}}\left(k_{x}, k_{y}, z\right)=\mathbf{h}_{\eta_{0}} e^{-i \gamma_{0} z}
\end{aligned}
$$

onde $\gamma_{0}=\left[k_{0}^{2}-u^{2}\right]^{1 / 2}$ e $k_{0}^{2}=\omega^{2} \mu_{0} \varepsilon_{0}$. A solução que representa uma onda eletromagnética que se propaga na direção dos : negativos foi eliminada uma vez que a região é ilimitada para $z>d_{N}$. Em conseqüência, as expressões para as componentes dos campos eletromagnéticos no domínio espacial são calculadas por:

$$
\begin{aligned}
& E_{\eta 0}=\frac{1}{4 \pi^{2}} \int_{-\infty}^{+\infty} \int_{\eta_{0}} e^{-i\left(k_{x} x+k_{y} y^{\prime}+\gamma_{0}=\right)} d k_{x} d k_{y} \\
& H_{\eta 0}=\frac{1}{4 \pi^{2}} \int_{-\infty}^{+\infty} \int_{\eta_{0}} e^{-i\left(k_{x} x+k_{y} y+\gamma_{0}-\right)} d k_{x} d k_{y}
\end{aligned}
$$

Também nesse caso, as equações de Maxwell permitem escrever as componentes $\mathbf{e}_{x 0}, \mathbf{e}_{y 0}, \mathbf{h}_{x o}$ e $\mathbf{h}_{y_{0}}$ como funções de $\mathbf{e}_{-0}$ e $\mathbf{h}_{70}$. como se segue:

$$
\begin{aligned}
& \mathbf{e}_{x 0}=u^{-2}\left(-\gamma_{0} k_{x} \mathbf{e}_{z 0}-\omega \mu_{0} k_{y} \mathbf{h}_{z 0}\right) \\
& \mathbf{e}_{y 0}=u^{-2}\left(-\gamma_{0} k_{y} \mathbf{e}_{z 0}+\omega \mu_{0} k_{x} \mathbf{h}_{z 0}\right) \\
& \mathbf{h}_{x 0}=u^{-2}\left(\omega \varepsilon_{0} k_{y} \mathbf{e}_{z 0}-\gamma_{0} k_{x} \mathbf{h}_{z 0}\right) \\
& \mathbf{h}_{y 0}=u^{-2}\left(-\omega \varepsilon_{0} k_{x} \mathbf{e}_{z 0}-\gamma_{0} k_{y} \mathbf{h}_{z 0}\right)
\end{aligned}
$$

onde $\mathbf{e}_{\eta 0}=\mathbf{e}_{\eta 0}\left(k_{x}, k_{y}\right)$ e $\mathbf{h}_{\eta 0}=\mathbf{h}_{\eta 0}\left(k_{x}, k_{y}\right)$. No espaço livre são desconhecidas as funções $\mathbf{e}_{-o}$ e $\mathbf{h}_{-0}$, resultando em um total de $4 N+2$ incógnitas a serem determinadas a partir da aplicação das condições de contorno pertinentes ao problema em questão.

\section{ESTRUTURAS ANALISADAS}

A versatilidade da estrutura apresentada na Figura 1 permite a análise de diversas geometrias de interesse da engenharia aeroespacial brasileira [6-8]. Neste trabalho, entretanto, serão abordados somente parâmetros elétricos relacionados com a antena de microfita retangular alimentada por uma prova coaxial e com a linha de fita que alimenta uma fenda retangular.

\subsection{ANTENA DE MICROFITA}

Na sua forma mais simples. a antena de microfita é constituída por um plano de terra e por um substrato dielétrico que suporta uma fita condutora. Tal geometria pode ser obtida da Figura 1 curto-circuitando-se a abertura e restrin-gindo as $N-T$ camadas a uma única camada de espessura $d$, como apresentado na Figura 2. A alimentação da antena é realizada por uma prova coaxial.

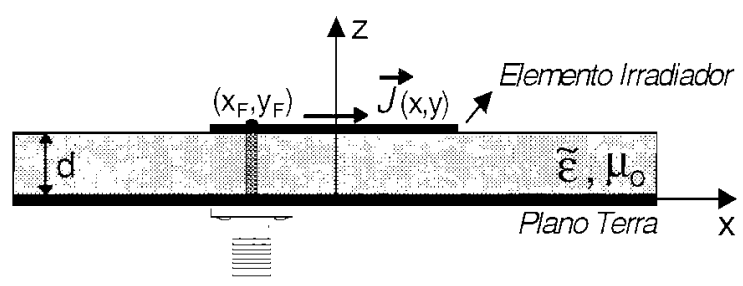

Figura 2. Antena de microfita.

De posse das equações (17), (18), (25) e (26) para os campos eletromagnéticos, as condições de contorno em $z=0$ (campo elétrico tangencial nulo ao longo do plano de terra, suposto um condutor perfeito) e em $z=d$ (continuidade da componente tangencial do campo elétrico e descontinuidade da componente tangencial do campo magnético), são aplicadas, resultando em um sistema de seis equaçôes nas incógnitas $\mathbf{e}_{z q}, \mathbf{h}_{z r}, \mathbf{e}_{-o}$ e $\mathbf{h}_{-0}$. Combinando convenientemente as soluções para os campos transformados, expressões fechadas e compactas para as funções de Green espectrais podem ser determinadas [5]. Exemplos dessas funções, para $z=d$, são apresentados abaixo,

$$
\begin{array}{r}
G_{X x}=\frac{i}{\omega u^{2}}\left[k_{x}^{2} \gamma_{0} \gamma_{2} T_{M}^{-1} \operatorname{sen}\left(\gamma_{2} d\right)\right. \\
\left.+k_{Y}^{2} k_{0}^{2} T_{E}^{-1} \operatorname{sen}\left(\gamma_{1} d\right)\right] \\
G_{x y}=\frac{i k_{x} k_{y}}{\omega u^{2}}\left[\gamma_{0} \gamma_{2} T_{M}^{-1} \operatorname{sen}\left(\gamma_{2} d\right)\right. \\
\left.-k_{0}^{2} T_{E}^{-1} \operatorname{sen}\left(\gamma_{1} d\right)\right]
\end{array}
$$

com

$$
\begin{aligned}
& T_{E}=\gamma_{1} \varepsilon_{0} \cos \left(\gamma_{1} d\right)+i \gamma_{0} \varepsilon_{0} \operatorname{sen}\left(\gamma_{1} d\right) \\
& T_{M}=\varepsilon_{.1} \gamma_{0} \cos \left(\gamma_{2} d\right)+i \varepsilon_{0} \gamma_{2} \operatorname{sen}\left(\gamma_{2} d\right) .
\end{aligned}
$$

onde $T_{E}$ e $T_{M}$ são as equações características dos respectivos modos de propagação $T E$ e $T M$ excitados no substrato, $G_{x x}$ é a componente da diádica de Green espectral que relaciona $\varepsilon_{x}$ com $j_{x}, G_{x y}$ é a componente da diádica que relaciona $\varepsilon_{x} \operatorname{com} j_{y}$ e $j_{\eta}=j_{\eta}\left(k_{x}, k_{y}\right)$ é a transformada de Fourier da componente $J_{n}(x, y)$ da densidade de corrente elétrica superficial.

Uma vez que o campo eletromagnético no domínio espacial é obtido através da transformada inversa de Fourier, integrais duplas nas variáveis espectrais devem ser calculadas. Entretanto, as densidades de corrente transformadas que aparecem em seus integrandos são, em geral, desconhecidas. Métodos numéricos, como o método dos momentos, têm sido utilizados na determinação dessas correntes. Como esses cálculos são complexos, uma análise criteriosa das funções de Green espectrais deve ser realizada. Por questões de espaço, somente os resultados relacionados com a função $G_{x}$, obtidos para $d=1,27 \mathrm{~mm}$ e $f=2,265 \mathrm{GHz}$ serão discutidos neste trabalho.

Uma análise da função complexa $G_{x x}$ nos revela que tal função é par nas variáveis espectrais $k_{k}$ e $k_{y}$, e pode apresentar um ou mais pólos. Consequentemente, cuidados especiais são necessários quando de sua integração. Para melhor 
visualizar o comportamento de $G_{x x}$, gráficos tridimensionais para as partes real e imaginária, calculados com $\mathcal{E}_{r \mathrm{x}}=13,0 \mathrm{e}$ $\varepsilon_{r-}=10,2$, e a mesma tangente de perdas $(0.002)$ nessas duas direções, são apresentados nas Figuras 3 e 4 . Nota-se

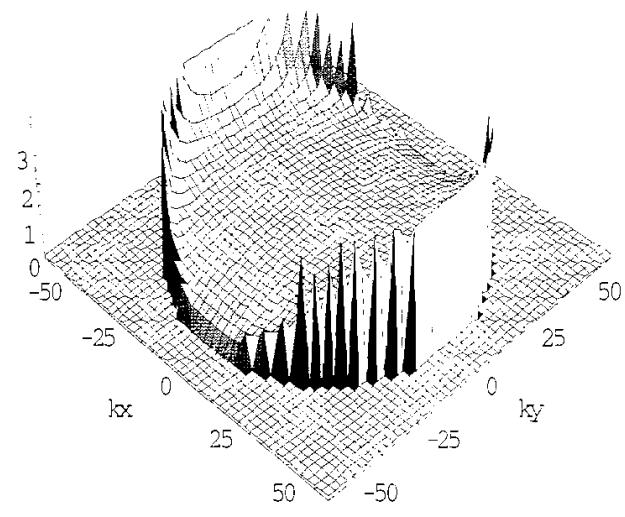

Figura 3. Gráfico tridimensional de $\operatorname{Re}\left[G_{x x}\right]$

desses gráficos que a função complexa $G_{x}$, no caso em questão, possui um único pólo. Este pólo é, na realidade, a constante de propagação do modo $T M_{0}$ que é excitado no substrato da antena. Numericamente, essa constante é determinada resolvendo-se a equaçâo $T_{M}(u)=0$.

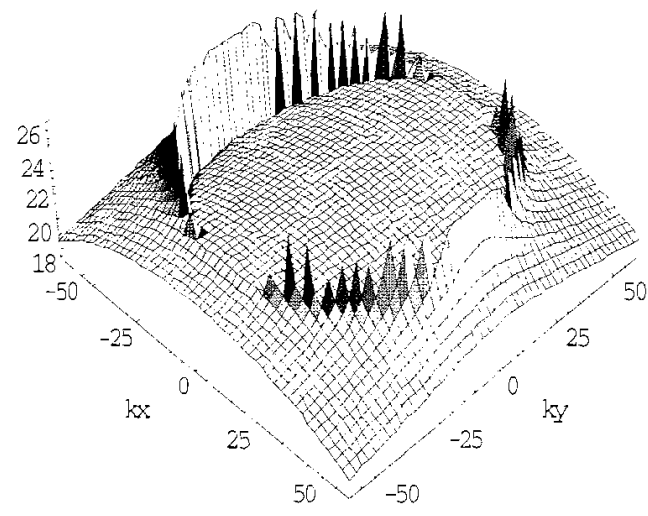

Figura 4. Gráfico tridimensional de $\operatorname{Im}\left[G_{x x}\right]$.

Conhecidas as funções de Green para a estrutura em questão, um sistema de equações integrais é estabelecido se a condição de campo elétrico tangencial nulo for imposta sobre o elemento irradiador (considerado um condutor perfeito). Seguindo o procedimento usual do método dos momentos (MoM) no domínio espectral e aplicando o método de Galerkin, que utiliza como função teste a própria função base, e os teoremas de Parseval e da reação [5], esse sistema integral é conduzido ao seguinte conjunto de $\left(N_{x}+N_{1}\right)$ equações lineares:

$$
\begin{aligned}
& \sum_{m=1}^{N_{x}} I_{m} Z_{p m}^{x x}+\sum_{n=1}^{N_{y}} I_{n} Z_{p n}^{\lambda y}=V_{p} \quad ; p=1, \ldots \ldots, N_{x} \\
& \sum_{m=1}^{N_{x}} I_{m} Z_{q m}^{y x}+\sum_{n=1}^{N_{y}} I_{n} Z_{q y}^{y \prime}=V_{q} \quad ; q=1, \ldots \ldots, N_{y}
\end{aligned}
$$

com

$$
\begin{aligned}
& Z_{p m}^{x u}=\frac{(-1)}{4 \pi^{2}} \int_{-\infty}^{+\infty} \int_{x} G_{x} j_{p}^{*} j_{m} d k_{x} d k_{y} \\
& Z_{p h}^{n y}=\frac{(-1)}{4 \pi^{2}} \int_{-\infty}^{+\infty} \int_{x y} j_{p} j_{n} d k_{x} d k_{y} \\
& Z_{i j m}^{v x}=\frac{(-j)}{4 \pi^{2}} \int_{-\infty}^{+\infty} \int_{y x} j_{y}^{*} j_{m} d k_{x} d k_{y} \\
& Z_{q i}^{i x}=\frac{(-1)}{4 \pi^{2}} \int_{-\infty}^{+\infty} \int_{y} G_{y} j_{q}^{*} j_{n} d k_{y} d k_{y} \\
& V_{p}=\iiint_{V_{f}} E_{p x}(x, y, z) \cdot \stackrel{\rho}{J}_{f}(x, y, z) d x d y d z \\
& V_{p}=\iiint_{V_{f}} E_{q y}(x, y, z) \cdot J_{f}(x, y, z) d x d y d z
\end{aligned}
$$

onde $\stackrel{\rho}{E}_{p x}(x, y, z)$ é o campo devido à densidade de corrente $J_{p}(x, y) \hat{x}, \hat{E}_{q v}(x, y, z)$ é o campo devido à densidade de corrente $J_{q}(x, y) \hat{y}, V_{f}$ é o volume do sistema de aljmentação que contém a densidade de corrente $\stackrel{\mu}{J}_{f}(x, y, z)$, $N_{x}$ e $N_{y}$ são os núnteros de modos nas direções $x$ e y e $I_{m}$ e $I_{i n}$ são os coeficientes numéricos das expansōes das densidades de corrente localizadas sobre o elemento irradiador. Como funções base foram utilizadas as de domínio completo, cujo formato é baseado nos modos de ressonância do método da cavidade [9].

O conector coaxial de alimentação, para antenas finas, pode ser modelado por uma prova de corrente constante, cuja densidade é dada por:

$$
\hat{J}_{f}(x, y, z)=\hat{z} I_{0} \delta\left(x-x_{f}\right) \delta\left(y-y_{f}\right)
$$

onde $I_{0}$ é a corrente na prova localizada nas coordenadas $x_{f}$ e $y_{f}$. Com esse modelo, fazendo $I_{0}=1 \mathrm{~A}$, a impedância de entrada da antena pode ser calculada pela seguinte expressão [5]:

$$
Z_{i n}=-\sum_{p=1}^{N_{x}} I_{p} V_{p}-\sum_{q=1}^{N_{y}} I_{q} V_{q}
$$

onde

$$
\begin{array}{r}
V_{p}=\frac{(-d)}{4 \pi^{2}} \frac{\left(\varepsilon_{x} \varepsilon_{z}^{-1}\right)}{\omega \varepsilon_{0}} \int_{-\infty}^{+\infty} \int \frac{\operatorname{sen}\left(\gamma_{2} d\right)}{\left(\gamma_{2} d\right)} \\
\frac{\gamma_{0} k_{x} j_{p}}{T_{M}} e^{-i\left(k_{x} x_{f}+k_{y} y_{f}\right)} d k_{x} d k_{y} \\
V_{q}=\frac{(-d)}{4 \pi^{2}} \frac{\left(\varepsilon_{x} \varepsilon_{z}^{-1}\right)}{\omega \varepsilon_{0}} \int_{-\infty}^{+\infty} \frac{\operatorname{sen}\left(\gamma_{2} d\right)}{\left(\gamma_{2} d\right)} \\
\cdot \frac{\gamma_{0} k_{y} j_{q}}{T_{M}} e^{-i\left(k_{x} x_{f}+k_{y} y_{f}\right)} d k_{x} d k_{y} .
\end{array}
$$

Para levar em conta a reatância da prova deve-se somar $+i X_{p}$ à impedância calculada por (44), sendo $X_{p}$ dada por [5]: 


$$
X_{p}=60 d k_{0} \ln \left[2 /\left(k_{0} d_{p} \sqrt{\varepsilon_{r}}\right)\right]
$$

onde $d_{p}$ é o diâmetro da prova coaxial de alimentação.

$\mathrm{Na}$ solução do sistema de equações, deparamos com integrais do tipo das de Sommerfeld que, em geral, são computacionalmente ineficientes. Para melhorar a eficiência do cálculo numérico dessas integrais, três técnicas foram utilizadas: redução ao primeiro quadrante, mudança de variável $\left[k_{x}=\beta \cos (\alpha)\right.$ e $\left.k_{r}=\beta \operatorname{sen}(\alpha)\right]$ e subtração do termo dominante. Por exemplo, a expressão (37), após a aplicação dessas técnicas, toma a seguinte forma:

$$
\begin{gathered}
Z_{p m}^{\alpha x}=C_{1} \int_{\beta=0}^{\infty} \int_{\alpha=0}^{\pi / 2}\left(G B_{1} \cos ^{2}(\alpha)+G B_{2} \operatorname{sen}^{2}(\alpha)\right) \\
\cdot R_{p m}^{x x} d \alpha d \beta+C_{2} I_{p m}^{x x}
\end{gathered}
$$

onde

$$
\begin{aligned}
& G B_{1}=\beta \varepsilon_{0} \gamma_{0} \gamma_{2}\left[\operatorname{sen}\left(\gamma_{2} d\right) / T_{M}\right]-G A_{1} \\
& G A_{1}=-\frac{\beta^{2} \sqrt{\varepsilon_{r x x} / \varepsilon_{r-x}}}{\varepsilon_{r x}+\sqrt{\varepsilon_{r x} / \varepsilon_{r z}}} \\
& G B_{2}=\beta \varepsilon_{0} k_{0}\left[\operatorname{sen}\left(\gamma_{1} d\right) / T_{E}\right] \\
& C_{2}=\frac{-i}{\pi^{2} \omega \varepsilon_{0}} \cdot \frac{\sqrt{\varepsilon_{r x x} / \varepsilon_{r z}}}{\varepsilon_{r x}+\sqrt{\varepsilon_{r x} / \varepsilon_{r z}}} \\
& I_{Y m m}^{x x}=\int_{\beta=0}^{\pi / 2} \int_{\alpha=0}^{\pi / 2}\left[\beta^{2} \cos ^{2}(\alpha)\right] R_{p m}^{x x} d \alpha d \beta \\
& R_{p m}^{x x}=\operatorname{Re}\left[j_{p x}^{*}\left(k_{x}\right) j_{m x x}\left(k_{x}\right)\right] \operatorname{Re}\left[j_{p y}^{*}\left(k_{y}\right) j_{m y \cdot}\left(k_{y}\right)\right](54) \\
& j_{l l}\left(k_{x}, k_{y}\right)=j_{l x x}\left(k_{x}\right) \cdot j_{l y y}\left(k_{y}\right)
\end{aligned}
$$

$\operatorname{com} h=m, n, p$ ou $q$.

Empregando a teoria acima descrita, foi desenvolvido um programa de computador (ANISOMSA) em FORTRAN 77 que calcula os coeficientes da expansão da densidade de corrente no elemento irradiador, a impedância de entrada da antena e a sua freqüência de ressonância. O programa analisa antenas retangulares (alimentadas por provas coaxiais) com substratos uniaxiais ou isotrópicos. As perdas são consideradas através da tangente de perdas do dielétrico.

A inclusão das perdas do substrato na formulação apresentada desloca o(s) pólo(s) da(s) onda(s) de superfície do eixo real, de forma que o cálculo das integrais duplas pode ser efetuado sem maiores problemas desde que, na integração em $\beta$, leve-se em conta que nas vizinhanças do(s) pólo(s) (com a variável de integração sobre o eixo real), a função a ser integrada varia rapidamente. Com o objetivo de minimizar o tempo de computação, os fatores comuns nos integrandos dos elementos da matriz do método dos momentos são calculados e armazenados para uso subsequiente. O mesmo é feito com as integrais independentes da freqüência.

Para ilustrar os resultados obtidos pelo programa, na Figura 5 são apresentados gráficos para as partes real e 54 imaginária da impedância de entrada de uma antena retangular. com as seguintes dimensões e parâmetros elétricos: $L=20,0 \mathrm{~mm}$ (dimensão ressonante), $W=30,0 \mathrm{~mm}, x_{f}=3,5$ $\mathrm{mm}, y_{f}=0,0 \mathrm{~mm}, d=1,27 \mathrm{~mm}, \varepsilon_{r x}=13,0, \varepsilon_{r x}=10,2 \mathrm{e}$ tangente de perdas igual a 0,002 . Para ilustrar o efeito da anisotropia, na mesma figura são apresentados os gráficos para um substrato isotrópico $\left(\varepsilon_{r:}=\varepsilon_{r z}=10,2\right)$. Como se pode notar, o efeito da anisotropia na forma da impedância de entrada da antena em questão não é significativo.

Freqüência de ressonância é outro parâmetro de fundamental importância no projeto de antenas de microfita, pois trata-se de um irradiador faixa estreita, usualmente da ordem de 1 a $2 \%$ para antenas eletricamente finas. Além disso, é conveniente mencionar que podem ser definidas pelo menos três freqüências de ressonância para tal antena, ou seja: a freqüência na qual a parte resistiva da impedância de entrada é máxima, a freqüência na qual a reatância é nula e a frequiência na qual o módulo do coeficiente de reflexão é mínimo. Como exemplo de aplicação dessa formulação a

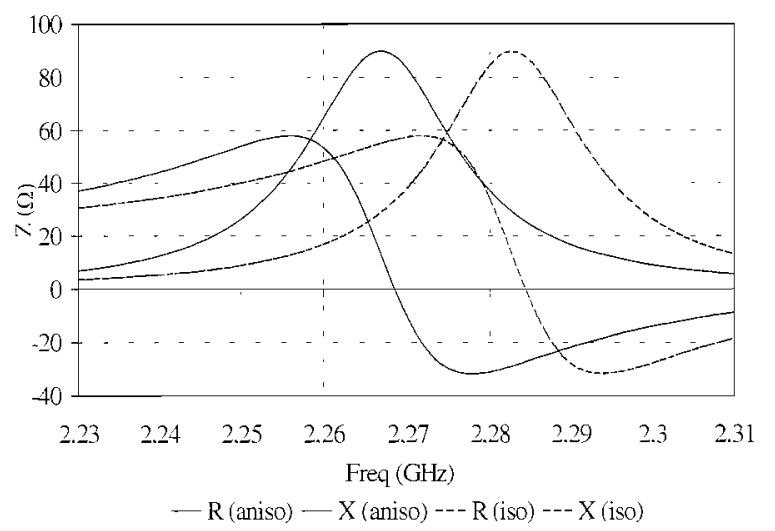

Figura 5. Gráficos para a impedância de entrada de uma antena de microfita retangular, calculados pelo programa ANISOMSA.

uma antena com substrato uniaxial, a Tabela 1 apresenta os valores calculados para a freqüência de ressonância (reatância nula) da antena retangular descrita acima, em comparação com o resultado experimental apresentado em [10]. Tal antena teria sido confeccionada com o substrato EPSILAM10, que é um material reconhecidamente anisotrópico [3]. Observa-se da Tabela 1 que o cálculo através do formalismo uniaxial resultou ser mais próximo do valor medido para a freqüência de ressonância da antena.

\begin{tabular}{|c|c|c|}
\hline \multirow{2}{*}{$\begin{array}{c}\text { Freqüência de } \\
\text { Ressonância } \\
\text { Experimental [10] } \\
\text { (MHz) }\end{array}$} & \multicolumn{2}{|c|}{$\begin{array}{c}\text { Valores Calculados } \\
\text { (MHz) }\end{array}$} \\
\cline { 2 - 3 } & $\begin{array}{c}\text { Isotrópico } \\
\varepsilon_{r x}=\varepsilon_{r x}=10,2\end{array}$ & $\begin{array}{c}\text { Uniaxial } \\
\varepsilon_{r x}=13,0 \varepsilon_{r x}=10,2\end{array}$ \\
\hline 2264 & 2284 & 2268 \\
\hline
\end{tabular}

Tabela 1. Tabela comparativa entre frequiências de ressonância teóricas e experimental.

Na Figura 6 são apresentados resultados teóricos e experimentais para a impedância de entrada de uma antena retangular com as seguintes dimensões e parâmetros elétricos: $L=64,5 \mathrm{~mm}$ (dimensão ressonante), $W=78,2 \mathrm{~mm}, x_{f}=$ $11,25 \mathrm{~mm}, y_{f}=0,0 \mathrm{~mm}, d=1,57 \mathrm{~mm}$, substrato isotrópico 
$\operatorname{com} \varepsilon_{r}=2,33 \pm 0,02$ (tolerância especificada pelo fabricante) e tangente de perdas igual a 0,0012 . Os pontos experimentais foram medidos com auxílio do analisador de

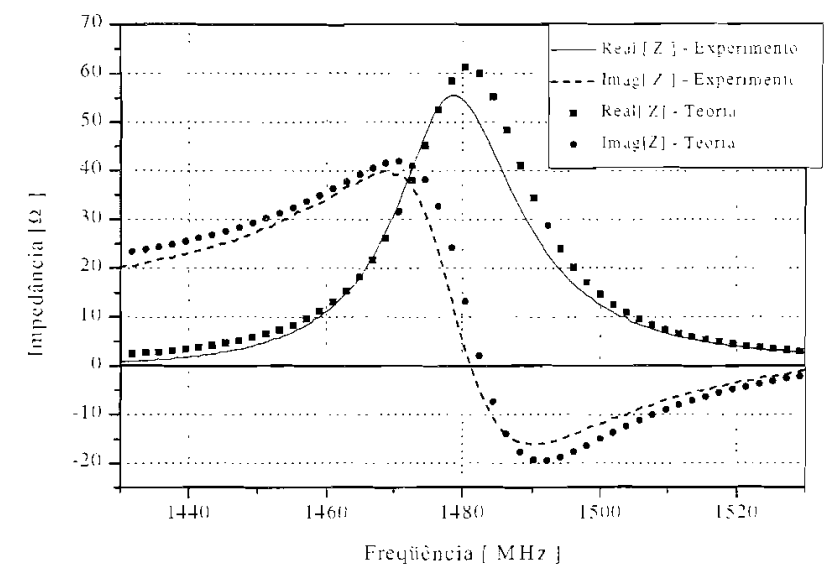

Figura 6. Impedância de entrada de uma antena de microfita retangular com substrato isotrópico.

redes HP 8714 C. A curva teórica foi obtida considerando a constante dielétrica do substrato igual a 2,35. portanto, dentro da tolerância do substrato. Nota-se dessa figura uma boa concordância entre os resultados teóricos e os experimentais. Por exemplo, para a frequiência na qual a resistência de entrada é máxima $\left(R_{m}\right)$ mediu-se $1478,5 \mathrm{MHz}$ contra $1480,5 \mathrm{MHz}$ calculado pelo programa ANISOMSA; isto é, um $\Delta f$ da ordem de $2,0 \mathrm{MHz}$. A mesma antena quando analisada pelo programa de simulação para estruturas multicamadas ENSEMBLE ${ }^{T M}$ [11] apresentou, no ponto onde a resistência é máxima, a freqüência de $1485 \mathrm{MHz}$ e um $\Delta f$ da ordem de $6,5 \mathrm{MHz}$. Também neste último caso utilizou-se $\varepsilon_{r}=2,35$. Os resultados obtidos pelo ANISOMSA e pelo ENSEMBLE $E^{T M}$ são equivalentes. com ligeira vantagem para o primeiro.

\subsection{LINHA DE FITA}

Outra geometria de interesse capaz de ser analisada como um caso particular da estrutura apresentada na Figura 1 é a antena de fenda retangular alimentada por uma linha de fita. Essa antena apresenta uma faixa de passagem maior que a da antena de microfita, além de uma melhor isolação entre a rede de alimentação e o elemento irradiador. A análise e o projeto de tais antenas é particularmente útil para o desenvolvimento de redes com feixes controlados eletronicamente, de grande interesse do setor aeroespacial.

Neste trabalho apresentaremos resultados preliminares obtidos, via método dos momentos, para a constante de propagação da linha de fita de alimentação da antena, de fundamental importância no cálculo de sua impedância de entrada. A geometria da linha de alimentação é um caso particular da apresentada na Figura 1 se a abertura localizada no plano $z=0$ for curto-circuitada e apenas duas camadas entre os planos condutores $(T=2)$ forem utilizadas (Figura 7).

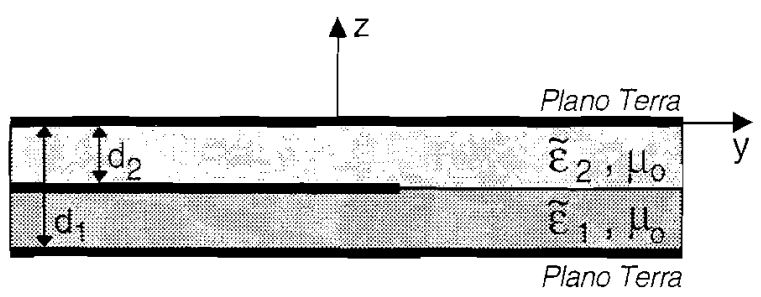

Figura 7. Linha de fita com anisotropia uniaxial.

De modo análogo ao desenvolvido no item anterior, aplicando-se as condições de contorno para os campos eletromagnéticos nas interfaces $z=-d_{2}$ (continuidade da componente tangencial do campo elétrico e descontinuidade da componente tangencial do campo magnético), $z=0$ e $z=-$ $d_{1}$ (campo elétrico tangencial nulo ao longo dos planos de terra, considerados condutores perfeitos), expressões para as funções de Green transformadas podem ser determina-das. Em particular. analisaremos a função $G_{n 11}$ que, na interface $z=-d_{2}$, é calculada pela seguinte expressão:

$$
\begin{aligned}
G_{y 11}= & \frac{-i \gamma_{21} \gamma_{22} k_{1}^{2} \operatorname{sen}\left(\gamma_{22} d_{2}\right) \operatorname{sen}\left[\gamma_{21}\left(d_{1}+d_{2}\right)\right]}{\omega u^{2} T_{M G}} \\
& -\frac{i \omega \mu_{0} k_{x}^{2} \operatorname{sen}\left(\gamma_{12} d_{2}\right) \operatorname{sen}\left[\gamma_{11}\left(d_{1}+d_{2}\right)\right]}{u^{2} T_{E G}}
\end{aligned}
$$

com

$$
\begin{aligned}
T_{E G}= & \gamma_{11} \operatorname{sen}\left(\gamma_{12} d_{2}\right) \cos \left[\gamma_{11}\left(d_{1}-d_{2}\right)\right] \\
& +\gamma_{12} \cos \left(\gamma_{12} d_{2}\right) \operatorname{sen}\left[\gamma_{11}\left(d_{1}-d_{2}\right)\right] \\
T_{M G}= & \varepsilon_{x 1} \gamma_{22} \operatorname{sen}\left(\gamma_{22} d_{2}\right) \cos \left[\gamma_{21}\left(d_{1}-d_{2}\right)\right] \\
& +\varepsilon_{x 2} \gamma_{21} \cos \left(\gamma_{22} d_{2}\right) \operatorname{sen}\left[\gamma_{21}\left(d_{1}-d_{2}\right)\right] .
\end{aligned}
$$

onde $T_{E G}$ e $T_{M G}$ são as equações características dos respectivos modos de propagação TE e $T M$ excitados nas camadas uniaxiais localizadas entre os planos condutores.

Gráficos tridimensionais para as partes real e imaginária de $G_{y 11}$, calculados com $d_{1}=3,524 \mathrm{~mm}, d_{2}=1,524 \mathrm{~mm}, f=$ $2,0 \mathrm{GHz}, \varepsilon_{r x]}=2,2 . \varepsilon_{r: 1}=2,4, \varepsilon_{r x 2}=3,2, \varepsilon_{r-2}=3,4$ e a mesma tangente de perdas $(0.0018)$ nessas direções, săo apresentados nas Figuras 8 e 9 .

Também neste caso, a função complexa $G_{y y 11}$ apresenta um único pólo, que está relacionado com a constante de propagação do modo confinado nos dielétricos uniaxiais localizados entre os planos de terra.

Usando as equações (57) e (58), curvas de dispersão para os modos confinados, calculadas nas mesmas condiçōes acima, porém para dielétricos sem perdas, são apresentadas na Figura 10. Pode-se observar dessas curvas que o modo fundamental de propagação não tem frequiência de corte e é não-dispersivo (modo quase-TEM). Por outro lado, a primeira frequiência de corte dos modos TE e TM é da ordem de $26.5 \mathrm{GHz}$. 


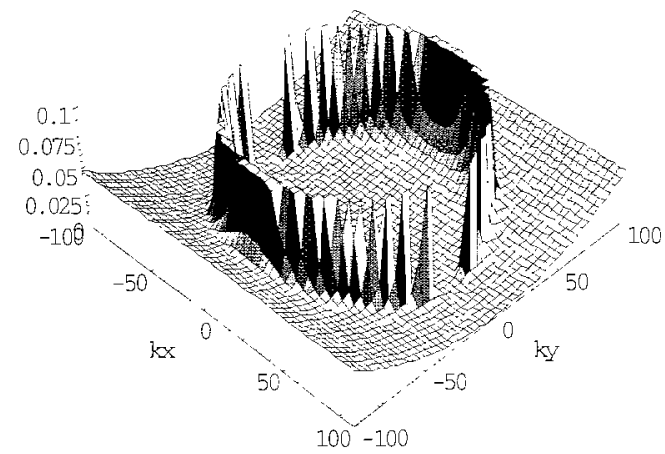

Figura 8. Gráfico tridimensional de $\operatorname{Re}\left[G_{y_{11}}\right]$.

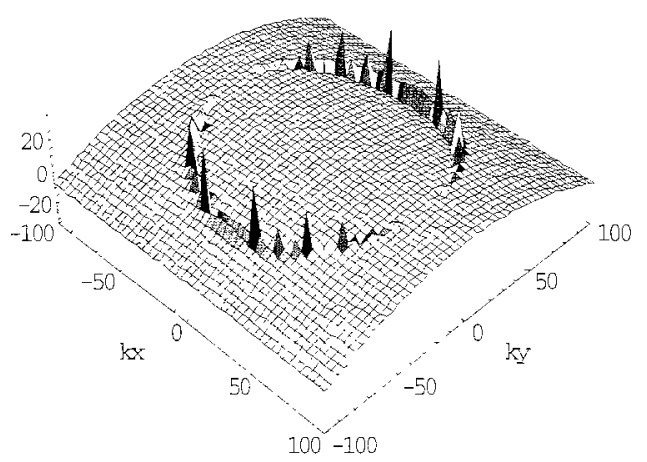

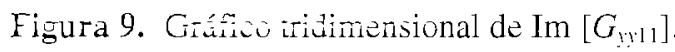

Como o modo fundamental confinado, de constante de propagação $k_{f c}$, é quase-TEM, é importante verificar se o mesmo pode ser excitado por uma linha de fita infinita. Para tanto, vamos calcular a constante de propagação $k_{e}$ do modo guiado por uma linha de fita infinitamente longa na direção $\therefore$ A densidade de corrente elétrica nessa linha, por conveniência, será considerada como o produto de duas funções independentes de $x$ e $y$ [12], de modo que

$$
J_{E}(x, y)=J_{E x}(x) e^{-i k_{e} y}
$$

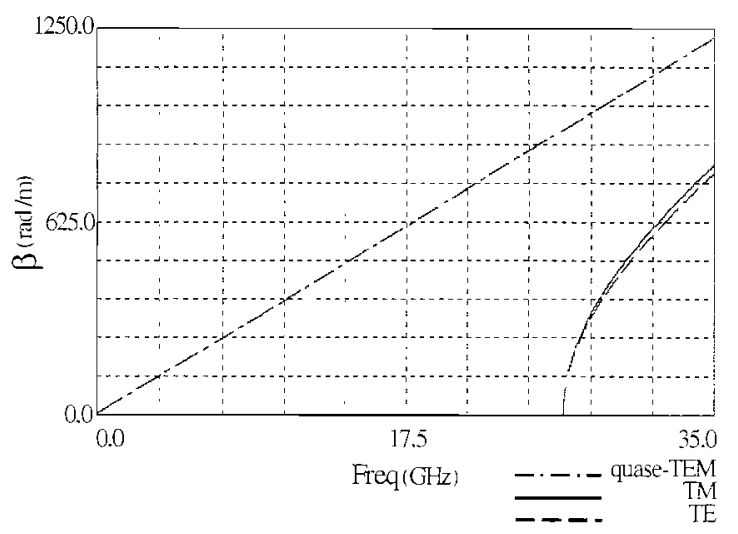

Figura 10. Curvas de dispersão para os modos confinados.
Utilizando a condição de componente tangencial nula para o campo elétrico ao longo da fita de largura $W_{L}$, considerada um condutor perfeito, obtém-se a seguinte equação integral em $k_{r}$ :

$$
\int_{-\infty}^{+\infty} G_{y, 11}\left(k_{x}, k_{e},-d_{2}\right)\left|j_{E x}\left(k_{x}\right)\right|^{2} d k_{x}=0 .
$$

Aplicando-se o método dos momentos na equação acima, valores numéricos para a constante de propagação $k_{e}$ podem ser determinados. Na Figura 11 são apresentados os resultados calculados para $W_{L}=5,6 \mathrm{~mm}, d_{1}=3,524 \mathrm{~mm}$ e $d_{2}=1,524 \mathrm{~mm}$, supondo-se a linha sem perdas e uma distribuição uniforme para a densidade de corrente $J_{E r}(x)$. A linha contínua descreve o comportamento da constante de propagação $k_{e}$, no caso de uma linha de fita com o seguinte grau de anisotropia para os dielétricos confinados: $\varepsilon_{i * 1}=2,2$, $\varepsilon_{r * 1}=2,4, \varepsilon_{r 22}=3,2$ e $\varepsilon_{r: 2}=3,4$. Em contrapartida, os pontos foram determinados considerando-se a seguinte condição de isotropia: $\varepsilon_{r \times 1}=\varepsilon_{r z 1}=1,0$ e $\varepsilon_{r 22}=\varepsilon_{r=2}=2,55$. Nota-se que esses pontos estāo muito próximos dos resul-tados previstos pelo programa de simulação para estruturas multicamadas ENSEMBLE TM [11].

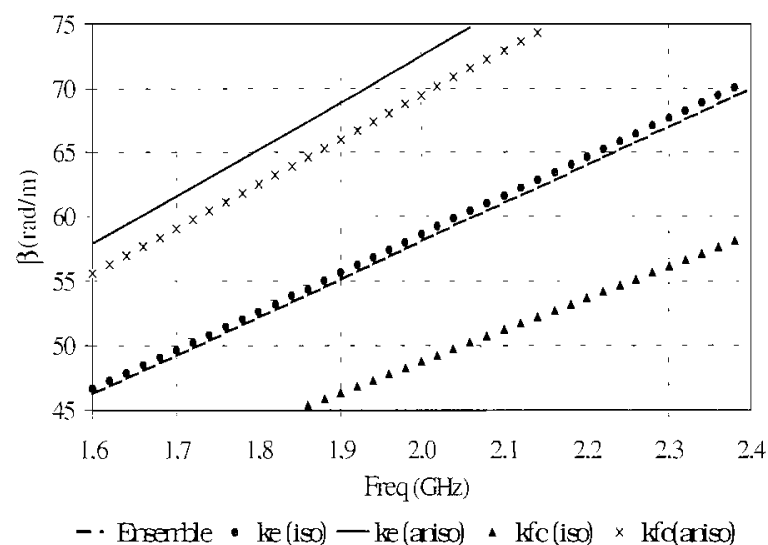

Figura 11. Curvas de dispersão para comparações de $k_{e}$ $\operatorname{com} k_{f c}$.

A constante de propagação do modo fundamental confinado $k_{\tilde{f}}$, obtido nas mesmas condições apresentadas acima, também são mostrados na Figura 11. Observa-se desses resultados que os valores de $k_{f c}$ estão mais próximos dos determinados para $k_{e}$, no caso de substratos anisotrópicos, que os obtidos na condição isotrópica. Entretanto, o valor de $k_{f c}$ é sempre menor do que o calculado para $k_{e}$, nas mes-mas condições. Isso demostra a impossibilidade da linha de fita de comprimento infinito excitar o modo de propagação fundamental confinado nos dielétricos localizados entre seus planos de terra. Por outro lado, se a linha de fita possui um comprimento finito e, portanto, está abruptamente terminada (Figura 1), ondas incidente e refletida coexistirão sobre a linha. Para esta situação, a densidade de corrente elétrica ao longo da direção y pode ser considerada como a superposição das ondas incidente e refletida, no modo fun- 
damental de propagação da linha, e de um número $M_{\mathrm{y}}$ de funções triangulares definidas a partir do extremo dessa linha. Além disso, a expansão ao longo da direção transversal $x$ deve levar em conta o fato dessa densidade de corrente tender a infinito nas bordas localizadas em $x=-W_{L} / 2$ e $x=+W_{L} / 2$ conhecido como condicão de borda. Na Figura 12 são apresentados os gráficos dos modos de expansão da densidade superficial de corrente elétrica $J_{E Y}(v)$, onde $L_{L}$ é o domínio da função triangular.

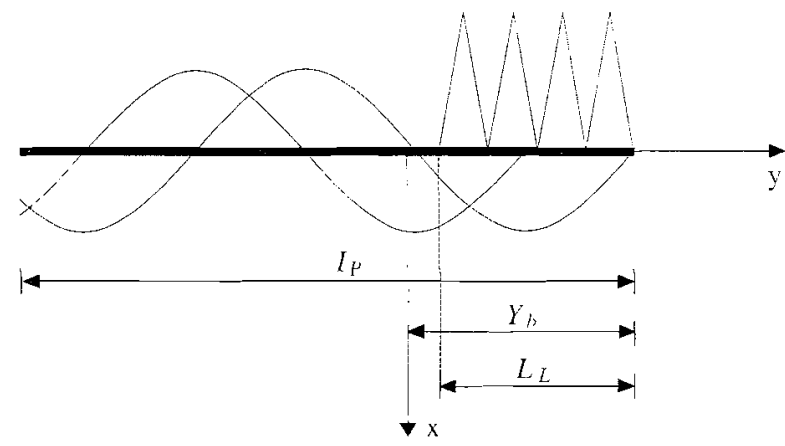

Figura 12. Modos de expansão da densidade de corrente $J_{E,}(y)$ sobre a linha de alimentação.

Para a linha de fita com as características elétricas e dimensões geométricas mostradas na Tabela 2, apresenta-se na Figura 13 o gráfico tridimensional da função densidade de corrente elétrica, calculada na freqüência de $2,0 \mathrm{GHz}$. Como esperado, nota-se a existência de uma onda estacionária na linha de fita com elevado coeficiente de reflexão.

\begin{tabular}{|c|c|c|c|}
\hline 1.1.1.1.1 & $\operatorname{Re}\left[\varepsilon_{r}\right]$ & $\begin{array}{c}\text { Tangente de } \\
\text { Perdas }\end{array}$ & $\begin{array}{c}\text { Espessura } \\
(\mathrm{mm})\end{array}$ \\
\hline 1 & 1,0 & 0,0 & 2,0 \\
\hline 2 & 2,55 & 0,0018 & 1,524 \\
\hline \multicolumn{4}{|c|}{ Dimensões (mm) } \\
\hline \multicolumn{2}{|c|}{$\overline{Y_{b}}$} & \multicolumn{2}{|c|}{8,8} \\
\hline \multicolumn{2}{|c|}{$I_{P}$} & \multicolumn{2}{|c|}{110,55} \\
\hline \multicolumn{2}{|c|}{$W_{L}$} & \multicolumn{2}{|c|}{5,6} \\
\hline
\end{tabular}

Tabela 2. Características elétricas e dimensões geométricas de uma linha de fita com substrato isotrópico.

Para melhor visualizar este fenômeno, na Figura 14 é apresentado o gráfico bidimensional dessa densidade de corrente, calculada no plano $x=0$. O coeficiente de reflexão, determinado numericamente pelo programa é, neste caso, complexo e vale: $\Gamma=0,8227+i 0,0198$. Consequentemente, $67 \%$ da potência incidente é refletida pela terminação abrupta da linha enquanto que $33 \%$ dessa potência é transferida ao substrato. Portanto, para as condições da linha da Tabela 2, o modo quase-TEM de substrato é fortemente excitado, transportando aproximadamente $1 / 3$ da potência incidente. Além disso, no diagrama de onda estacionária apresentado na Figura 14 nota-se que o comprimento de onda do modo guiado pela linha de fita, na frequiência de 2,0 GHz, é da ordem do comprimento físico da linha.

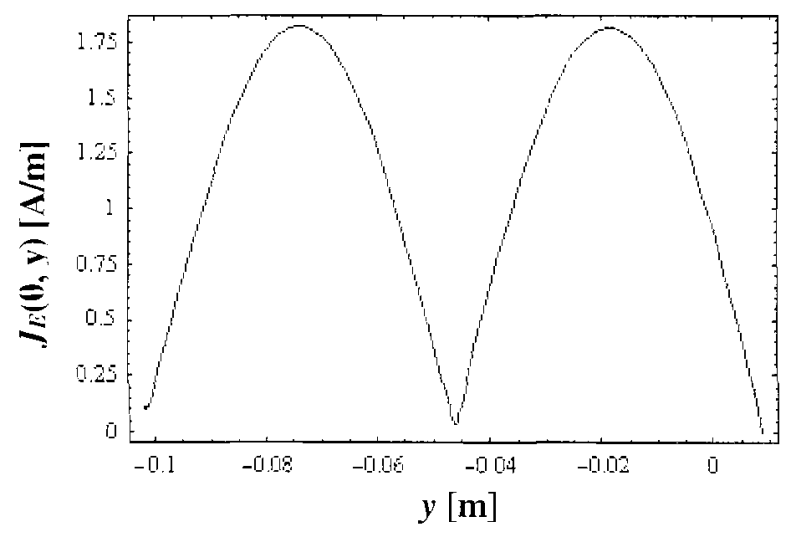

Figura 13. Gráfico 3D da densidade de corrente elétrica sobre a linha de fita definida na Tabela 2.

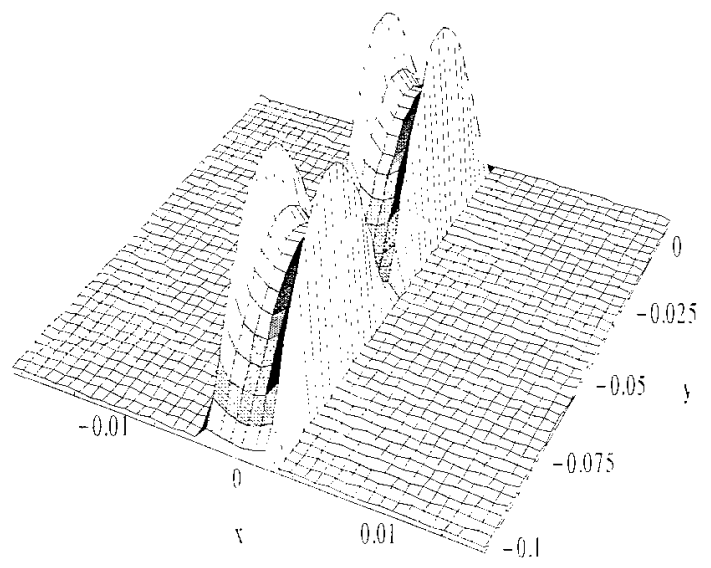

Figura 14. Gráfico 2D da densidade de corrente elétrica sobre a linha de fita com substrato isotrópico.

\section{COMENTÁRIOS E CONCLUSÕES}

Usando o formalismo de Fourier, no presente trabalho é apresentado um modelo para análise de estruturas planas multicamadas uniaxiais. São discutidas duas geometrias de interesse do setor aeroespacial; a antena de microfita retangular e a linha de fita que alimenta uma antena de fenda retangular. Como os campos eletromagnéticos nas diversas camadas são determinados através de integrais duplas de Fourier, funções de Green espectrais puderam ser calculadas. Gráficos tridimensionais foram traçados para auxiliar no estudo do comportamento dessas funções.

Embora o efeito da anisotropia na impedância de entrada da antena de microfita analisada não tenha sido significativo, sobre a freqüência de ressonância seu efeito não é desprezível, principalmente no caso de antenas eletricamente finas 
No caso da linha de fita, os resultados numéricos para as constantes de propagação $k_{f}$ e $k_{e}$ mostraram a impossibilidade da linha de comprimento infinito excitar o modo de propagação fundamental confinado nos dielétricos localizados entre seus planos de terra. Por outro lado, se a linha possui um comprimento finito e, portanto, está abruptamente terminada, nossos cálculos mostraram que esse modo de propagação pode ser excitado. Esse resultado será de grande importância quando da montagem do método dos momentos para o cálculo da impedância de entrada da antena de fenda retangular.

\section{AGRADECIMENTOS}

Este trabalho foi realizado com apoio parcial da CAPES e da OACI/PNUD-BRA-006/92, Objetivo 6.

\section{REFERÊNCIAS}

[1] F. Mesa, R. Marqués, and M. Horno, "On the computa-tion of the complete spectral Green`s dyadic for layered bianisotropic structures," IEEE Trans. Microwave The-ory Tech., vol. 46, pp. 1158-1164, Aug. 1998.

[2] J. F. Nye, Physical properties of crystals. London: Oxford Press, 1957.

[3] N. G. Alexopoulos, "Integrated-circuit structures on ani-sotropic substrate," IEEE Trans. Microwave Theory Tech., vol. 33, pp. 847-881, Oct. 1985.

[4] C. Scott, The spectral domain method in electromagnet-ics. Norwood: Artech House, 1989.

[5] L. Cividanes, Análise de antenas de microlinha em estruturas multicamadas com dielétricos uniaxiais. Tese de Doutorado, ITA, 1992.

[6] J. C. S. Lacava, C. R. Pérez Rodrigues, \& C. C. M. Junqueira, "Uma solução para as redes de antenas conformáveis dos foguetes SONDA IV e VLS operarem com dois canais de telemetria em banda S," 7o Simpósio Brasileiro de Telecomunicações. Florianópolis, Anais: 332 335, set. 1989.

[7] F. Lumini, and J. C. S. Lacava, "Analysis and design of microstrip antennas for aircraft applications," Proceedings of SAE Brasil 92 - Mobility Technology Confer-ence \& Exhibit. São Paulo, Brazil, Oct. 1992 (technical paper 921471E-SAE).

[8] L. Cividanes, V. P. D. Costa Jr., e J. C. S. Lacava, "Antena de microlinha para microssatélite científico," 120 Simpósio Brasileiro de Telecomunicações. Águas de Lindóia, Anais, V-II: 542 -545, set. 1995.

[9] Y. T. Lo, D. Solomon, and W. F. Richards, "Theory and experiment on microstrip antennas," IEEE Trans. Antennas Propagat. vol. 27, pp. 137-145, Mar. 1979.

[10] D. M. Pozar, "Radiation and scattering from a microstrip patch on a uniaxial substrate," IEEE Trans. An-tennas Propagat. vol. 35, pp. 613-621, Jun. 1987.

[11] ENSEMBLETM - Version 4.02, "Design, review, and 1D array synthesis," Boulder Microwave Technolo-gjes, Inc., 1996.
[12] U. V. Gothelf, and A. Ostergaard, "Full-wave analysis of a two slot microstrip filter using a new algorithm for computation of the spectral integrals," IEEE Trans. Microwave Theory Tech., vol. 41, pp. 101-108, Jan. 1993.

Lucio Cividanes nasceu em Nova Friburgo, RJ, em 1953. Graduou-se em Engenharia Elétrica (especialização em Telecomunicações) pela Universidade Federal Fluminense em 1974. Obteve o título de Mestre em Engenharia Eletrônica e Telecomunicações em 1978 pelo Instituto Nacional de Pesquisas Espaciais (INPE) e Doutor em Ciências pelo Instituto Tecnológico de Aeronáutica (ITA) em 1992. Atua como engenheiro da Divisão de Eletrônica Aeroespacial do INPE, trabalhando no desenvolvimento de antenas e circuitos de microondas para satélites. Tem experiência internacional na ESA (European Space Agency), estagiando no European Space and Tecnology Center (ESTEC) na Holanda, e trabalhando na firma SPAR no Canadá. Atuou também no Satélite Sino-Brasileiro de Recursos Naturais (CBERS), lançado em 1999, tendo participado na China de diversas atividades de projeto, fabricação e testes desse satélite. Suas principais áreas de interesse são antenas de microfita, redes planares e circuitos associados para aplicação espacial. É membro da Sociedade Brasileira de Microondas e Optoeletrônica.

Ana Vanessa Proaño De la Torre nasceu em Quito, Equador, em 1971. Graduou-se em Engenharia Eletrônica pela Escuela Politécnica del Ejército, Equador, em 1996. Obteve o título de Mestre em Ciências pelo Instituto Tecnológico de Aeronáutica, São José dos Campos, em 1999. Desde 1996 é Engenheira de Projetos no Centro de Investigación Científica y Tecnológica del Ejército, Equador. Atualmente é Professora de Engenharia Eletrônica na Escuela Politécnica del Ejército.

José Carlos da Silva Lacava nasceu em Caçapava, SP, em 1951. Graduou-se em Engenharia Elétrica (opção Eletrônica) pela Faculdade de Engenharia de São José dos Campos em 1974. Obteve os títulos de Mestre em Engenharia Eletrônica (1979) e Doutor em Ciências (1985) pelo Instituto Tecnológico de Aeronáutica (ITA), São José dos Campos. É Professor Adjunto da Divisão de Engenharia Eletrônica e atual Chefe do Departamento de Microondas e Optoeletrônica do ITA. Entre 1986 e 1987 foi Pesquisador Adjunto do Instituto de Atividades Espaciais, desenvolvendo pesquisa em redes cilíndricas de antenas de microlinha para os canais de telemetria dos foguetes Sonda IV e VLS. De 1992 a 1993 foi Professor Associado Convidado da Universidade da Beira Interior, Covilhã, Portugal. Entre 1993 e 1994 foi Professor Assistente Doutor da Universidade Estadual Paulista "Júlio de Mesquita Filho" - Faculdade de Engenharia de Guaratinguetá. Teoria eletromagnética, dispositivos passivos em microondas e antenas são suas áreas de interesse. É membro da Sociedade Brasileira de Eletromagnetismo e da Sociedade Brasileira de Microondas e Optoeletrônica. 